\title{
Modelo de un sistema automático en detección y diagnóstico de fallas basado en redes de petri en el proceso hogar de la Empresa Termotasajero Colgener S.a. E.s.p.
}

Marlon Mauricio Hernández Cely ${ }^{1} \quad$ Freddy Alejandro Leal Gonzalez ${ }^{2}$

Recibido:

Agosto 30 de 2011

Aceptado:

Abril 18 de 2012
${ }^{1}$ IE. MSc, de la universidad de pamplona. Docente de la Universidad Francisco de Paula Santander (UFPS). Semillero de Investigación en Detección y Diagnostico de Fallas en Sistemas de Automatización y Control Industrial (SIDDFA). Marlon25_3@hotmail.com

${ }^{2}$ IE de la Universidad Francisco de Paula Santander (UFPS),

Semillero de Investigación en Detección y Diagnostico de Fallas en Sistemas de Automatización y Control Industrial (SIDDFA). Freddy. alejandro.leal@hotmail.com

\section{Resumen}

Este artículo trata del diseño de un sistema automático en detección y diagnostico de fallas (SDDF) para el caso de la rotura de tubo en la empresa Termotasajero Colgener, el SDDF está basado en Redes de Petri e implementado en una tarjeta Spartan 2E de Xilinxs bajo la norma IEEE 10760/2008. La implementación en la tarjeta FPGA es precisa, factible y confiable utilizando el método experimental que se propone como punto inicial de programación en Redes de Petri con dispositivos FPGA y su aplicación en SDDF. Donde se exponen las ventajas de implementar la tecnología de las FPGA en el campo industrial.

Palabras clave: Spartan 2E de Xilinxs, Sistemas de Detección y Diagnostico de Fallas (SDDF), generador de vapor.

\section{Abstract}

This paper present the desing of an automatic system of detection and diagnostic of faults (SDDF) for the case of ruptured pipe in the company Termotasajero Colgener the SDDF is based on Petri Nets and implemented in a Spartan 2E of Xilinxs card under the IEEE 10760/2008. The implementation in FPGA card is precise, feasible and reliable using the experimental method which is proposed as starting point por Petri Nets programming FPGA with devices and their application in SDDF. Which displays the benefits of implementing the FPGA technology in the industrial sector.

Keywords: Spartan 2E de Xilinxs, Systems Fault Detection and Diagnosis (SDDF), steam generator. 


\section{Introducción}

E n el área de Sistemas de Detección y Diagnostico de Fallas (SDDF), cuentan con varias ramas de estudios, entre estas se encuentran el uso de técnicas de modelado como son la Máquinas de Estado Finitas [2-4], pero debido a su poca aplicabilidad y explosión combinacional en procesos de mediana complejidad ha hecho que otros investigadores enfoquen sus desarrollos utilizando las Redes de Petri [49]. En el trabajo aquí presentado, se realizó el modelado con Redes de Petri en el proceso hogar del generador de vapor (donde se lleva a cabo la combustión, mezcla de aire más carbón, generándose una bola de fuego tangencial para transmitir calor por medio de sus serpentines y poder convertir el agua de estado liquido a gaseoso ) de Termotasajero Colgener. El método desarrollado consiste en obtener un modelo de causalidad del elemento a diagnosticar, utilizando una red de Petri denominada red hacia adelante, [1][10].

Los elementos de arreglo de compuertas programables en campooField Programmable Gate Array, presentan una mayor velocidad de respuesta (secuencial y concurrente) con respectos a otros sistemas programables en el mercado, aunque en la actualidad cuentan con poca aplicabilidad en la industria debido a las condiciones hostiles que limitan su implementación.

El presente artículo pertenece a la Central Termoeléctrica Termotasajero Colgener S.a. E.s.p. ubicada en el municipio de San Cayetano Norte de Santander Colombia, a base de carbón, donde se presentaron fallas esporádicas en una caldera acuatubular que alimenta una turbina de $150 \mathrm{MW}$, causadas por la rotura de tubo.

\section{Metodología para el modelado del sistema}

La nomenclatura utilizada para este documento se presenta a continuación:

PSVS: Presión de Salida Vapor Sobrecalentado.

TSVS: Temperatura de Salida Vapor Sobrecalentado.

PES: Presión Entrada Sobrecalentador.

FVS: Flujo Vapor Sobrecalentador.

DPH: Alta Presión Hogar.

PDB: Presión Domo Baja.

NDB: Nivel Domo Bajo.

DCRT: Disparo Caldera Rotura Tubo

AAPH: Alarma Alta Presión Hogar

\subsection{Etapa I: Identificar el modelo causal del sistema a diagnosticar.}

La primera etapa en el proceso de diagnóstico, consiste en obtener un modelo causal en redes de Petri del proceso hogar del generador de vapor, en esta etapa del desarrollo, se utilizaron los modelos descriptivos como el que se ilustra en la figura 1.

Figura 1. Modelo causal del proceso en el hogar en una Red de Petri marcada hacia delante.

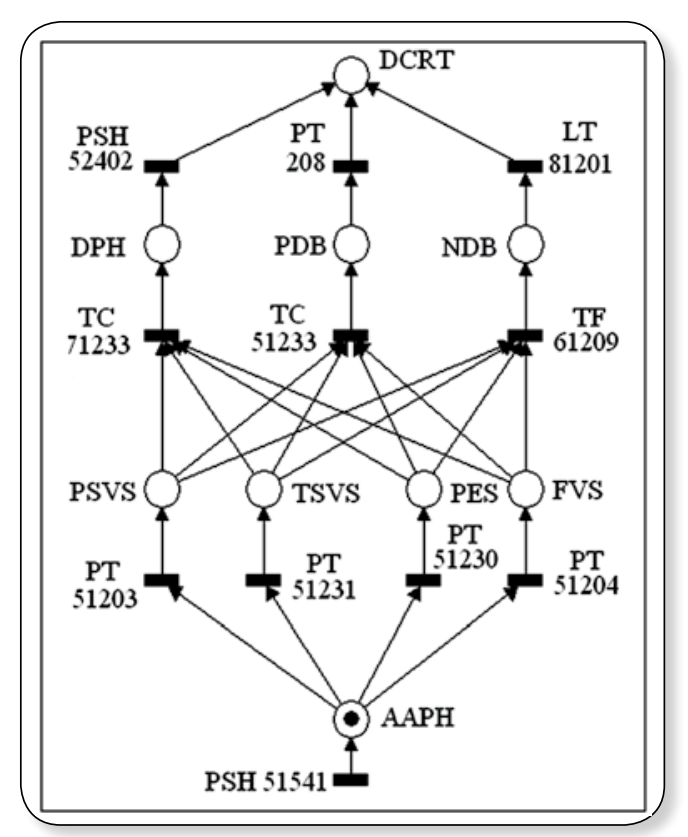


Modelo de un sistema automático en detección y diagnóstico de fallas basado en redes de petri en el

En el modelo de red de Petri, los P-elementos (lugares: DCRT, DPH, PDB, NDB, PSVS, TSVS, PES, FVS, AAPH) son formados por los elementos que intervienen en la falla y los T-Instrumentos (transiciones: PSH 52402, PT 208, LT 81201, TC 71233, TC 51233, TF 61209, PT 51203, PT51231, PT 51230, PSH 51541), fueron constituidos por la transición de la falla y el periodo de tiempo que ocurre entre la propagación de un elemento a otro, es decir, los umbrales se establecieron en las transiciones (instrumentos del hogar). Cuando la última transición se dispara, se establecerá la declaración de la falla [1].

\subsection{Etapa II: Diseño del modelo bajo} Redes de Petri para el Sistema de Detección y Diagnóstico de Fallas (SDDF) en el proceso Hogar.

A partir de los modelos causales de los elementos de la red de Petri de la figura 1, se obtuvieron los modelos de fallas, para esto, basto con invertir la dirección de los arcos de conexión de los elementos como se muestran en figura 2. Además, se incluyen en la red de diagnóstico la señal de alarma alta presión hogar (AAPH), que será de ayuda para la labor de diagnóstico.

Figura 2. Modelo causal del proceso en el hogar en una red de Petri marcada hacia atrás.

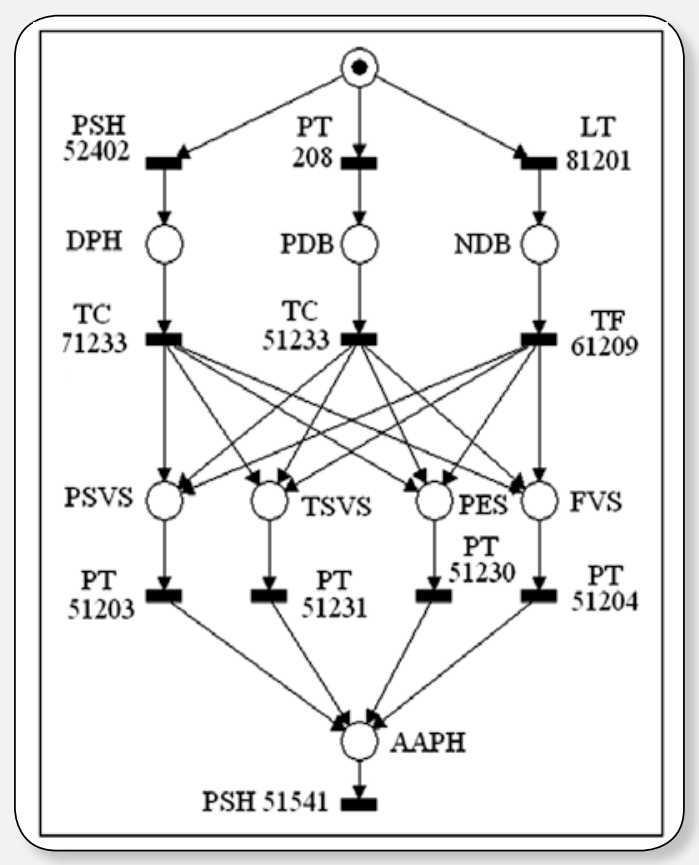

Debido a que no se cuenta con un modelo matemático, no se obtienen residuos para hacer diagnóstico, por lo que el sistema de diagnóstico basa su operación en alarmas tradicionales (superación de un umbral establecido) como son rangos de operación, que presentan los elementos (DCRT, DPH, PDB, NDB, PSVS, TSVS, PES, FVS), en el hogar. En dado caso que una variable sobrepase el umbral establecido, se indica un síntoma; para que se declare una falla, es necesario que todos los síntomas se presenten y de esta manera se descartan falsas alarmas.

\subsection{Etapa III: Simulación de la red} de Petri para el Sistema de Detección y Diagnostico de Fallas (SDDF) en el proceso Hogar.

Para la simulación de la falla se tendrán en cuenta los parámetros de operación y de alarma existentes en la caldera que se dan en la tabla 1. Además del SDDF, el proceso del hogar de generación de vapor se simuló en la toolbox de Matlab PNTool, donde se observa el estado de los lugares y el disparo de las transiciones a medida que pasa el tiempo tanto del lugar AAPH y la transición PSH 51541 hasta el lugar DCRT, y la transición PSH 52402, como se aprecia en la figura 3 y 4.

Tabla 1. Parámetros principales de disparo de alarma

\begin{tabular}{|c|c|}
\hline \multicolumn{2}{|c|}{$\begin{array}{c}\text { metros Principales de Disparo y Alarmas de la } \\
\text { Unidad }\end{array}$} \\
\hline $\begin{array}{l}\text { Alarma alto } \\
\text { nivel agua } \\
\text { Domo }\end{array}$ & $250 \mathrm{~mm}$. Por encima del N. N \\
\hline $\begin{array}{l}\text { Alarma bajo } \\
\text { nivel agua } \\
\text { Domo }\end{array}$ & $178 \mathrm{~mm}$.Por debajo del N. N \\
\hline $\begin{array}{l}\text { Corte bajo nivel } \\
\text { agua Domo }\end{array}$ & $280 \mathrm{~mm}$.Por debajo del N. N \\
\hline $\begin{array}{l}\text { Alarma alta } \\
\text { presión de hogar }\end{array}$ & $330 \mathrm{~mm} \cdot \mathrm{H}_{20}$ \\
\hline $\begin{array}{l}\text { Corte alta } \\
\text { presión del } \\
\text { hogar }\end{array}$ & $100 \mathrm{~mm} \cdot \mathrm{H}_{20}$ \\
\hline $\begin{array}{l}\text { Alama baja } \\
\text { presión de hogar }\end{array}$ & $-100 \mathrm{~mm} \cdot \mathrm{H}_{20}$ \\
\hline $\begin{array}{l}\text { Corte baja } \\
\text { presión de hogar }\end{array}$ & $-250 \mathrm{~mm} \cdot \mathrm{H}_{20}$ \\
\hline $\begin{array}{l}\text { Alarma alta } \\
\text { temp vapor } \\
\text { Sobre }\end{array}$ & $543^{\circ} \mathrm{C}$ \\
\hline
\end{tabular}


Respuestas

Año 17

No. 1

Junio 2012

ISSN 0122-820X
Figura 3. Simulación en PNToll de Matlab, del causal del proceso en el hogar simulado en una red de Petri marcada hacia adelante.

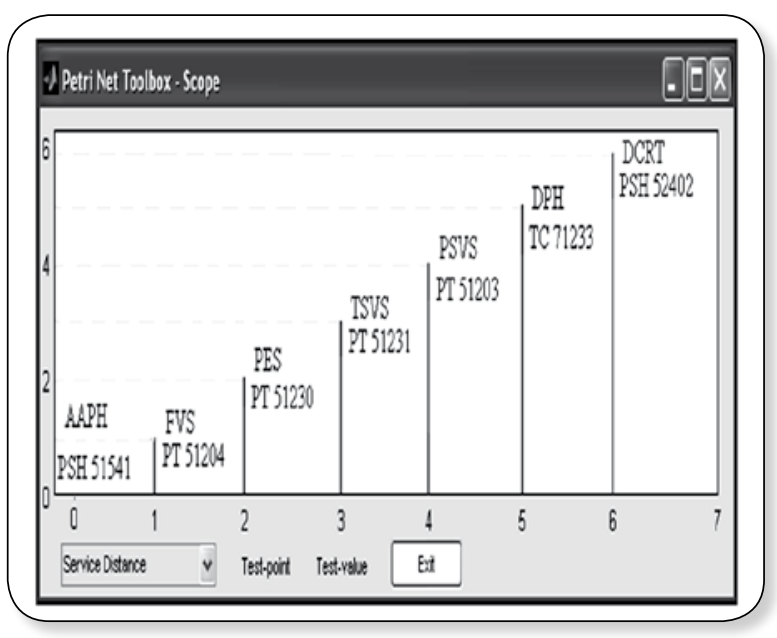

Figura 4. Modelo causal del proceso en el hogar simulado en una red de Petri marcada hacia adelante.

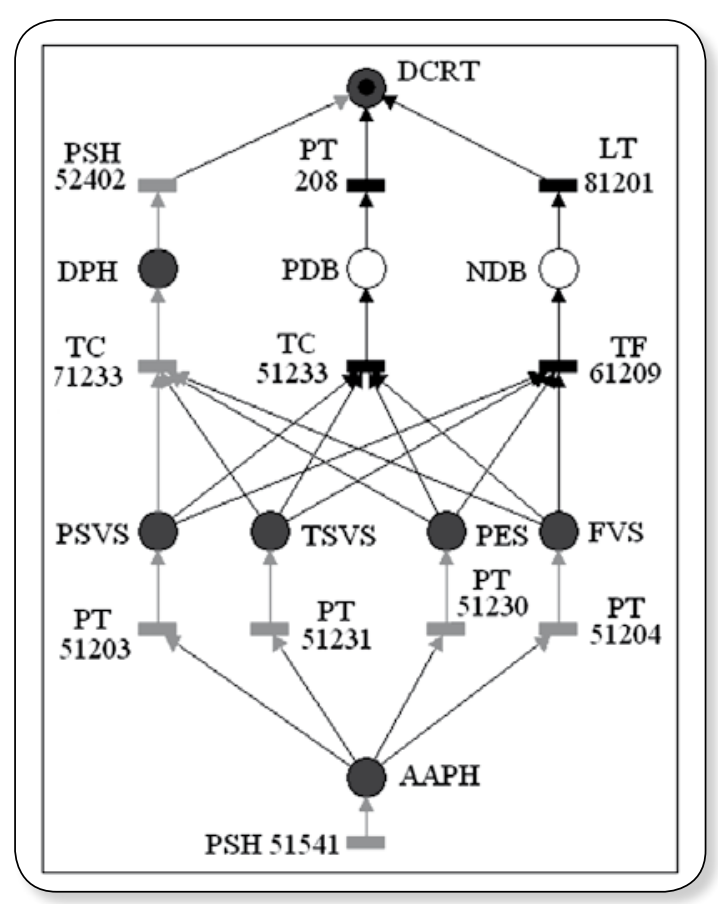

De igual forma se simuló la red de Petri en marcha hacia atrás en el toolbox de Matlab PNTool en donde se muestra el camino que recorre desde el lugar DCRT y la transición PSH 52502 hasta el lugar AAPH y la transición PSH 51541, que corresponde al mismo camino pero en dirección contraria, ver figuras 5 y 6 .
Figura 5. Simulación en PNToll de Matlab, del causal del proceso en el hogar simulado en una red de Petri marcada hacia atrás.

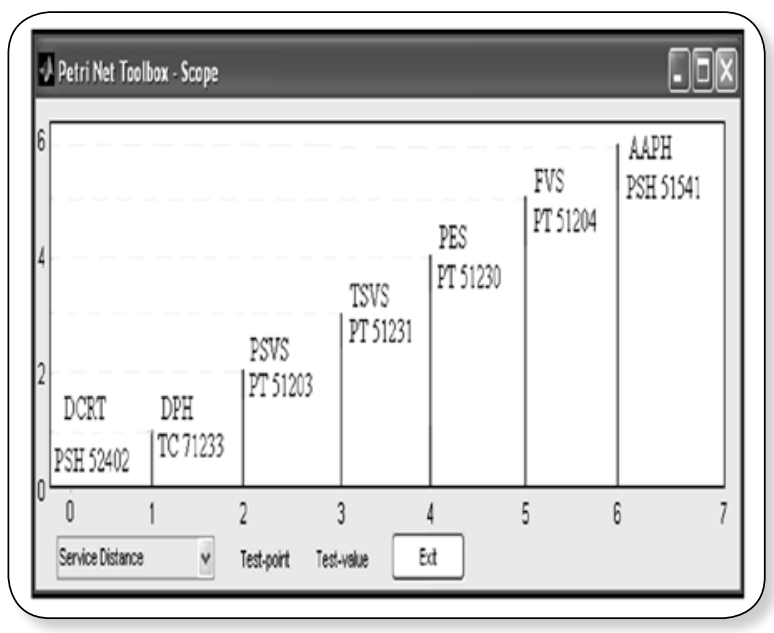

Figura 6. Modelo causal del proceso en el hogar simulado en una red de Petri marcada hacia atrás.

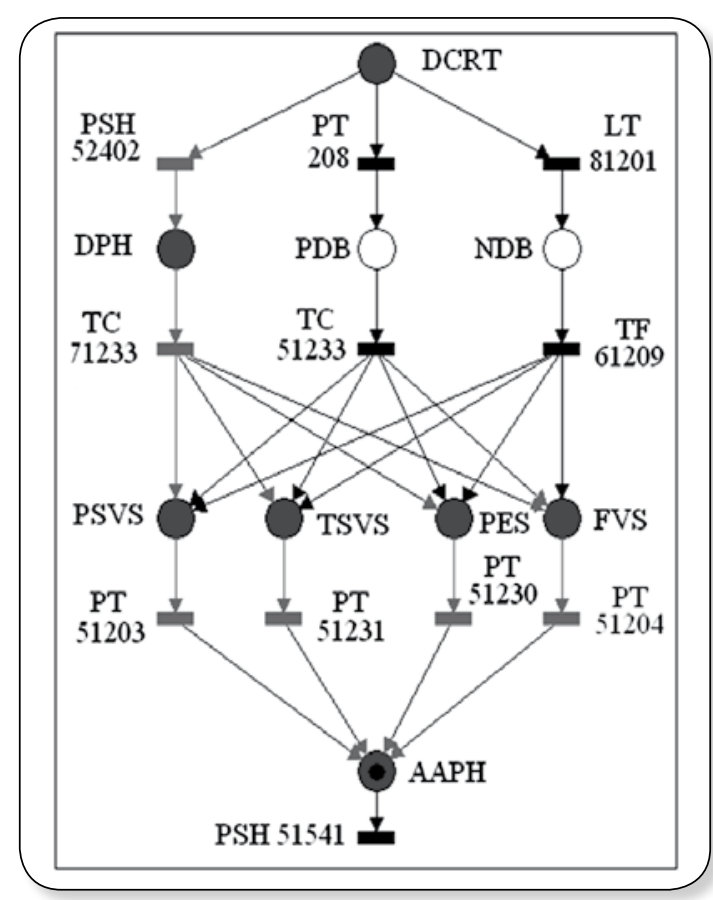

2.4. Etapa IV: Propuesta de Implementación Tecnológica del Sistema de Detección y Diagnostico de Fallas (SDDF), en el proceso Hogar.

La implementación tecnológica se efectúa con la tarjeta Spartan 2E de Xilinxs elegida por ser una herramienta de fácil adquisición y por su capacidad de procesamiento. 
Modelo de un sistema automático en detección y diagnóstico de fallas basado en redes de petri en el

La tarjeta Spartan requiere una alimentación de 5 Voltios en DC la cual viene incluida dentro del dispositivo por medio de un adaptador a $120 \mathrm{~V}$. la tarjeta cuenta con puertos entradas/salida. El dispositivo FPGA es programado en lenguaje VHDL por medio del software ISE Foundation de Xilinxs y programado sobre la norma IEEE estándar 1076 que define el VHSIC lenguaje de descripción de hardware o VHDL. La arquitectura del VHDL esta descrita de forma Behavioral (comportamiento) la cual se basa principalmente en el uso de procesos y de declaraciones secuenciales, las cuales permiten modelar la función con rapidez.

\section{Resultadosydiscusión}

Para la respectiva validación de la red de Petri para el SDDF se simulo en la toolbox de Matlab PNTool; y el programa implementado en VHDL- Behavioral bajo el estándar IEEE 1076/2008, se simulo en la aplicación Test Bench WaveFrom del software ISE Foundation de Xilinxs, con datos de operación reales según la tabla1, del proceso en el hogar del generador de vapor, además la secuencia de disparo de las transiciones y superación de los umbrales establecidos concuerdan con exactitud con la secuencia de operación en el hogar.
La gráfica paralela a la figura 5 y 6 simulada directamente del ISE Foundation de Xilinxs se muestra en la figura 7, en donde podemos apreciar la variación de la salida (lugares: DCRT, DPH, PDB, NDB, PSVS, TSVS, PES, FVS, AAPH) con respecto a las entradas actuales (transiciones: PSH 52402, PT 208, LT 81201, TC 71233, TC 51233, TF 61209, PT 51203, PT51231, PT 51230, PSH 51541) en el sistema dependiente del tiempo. Ver figura 7 .

\section{Conclusiones}

El sistema de diagnóstico en redes de Petri implementado permite una rápida detección e identificación de las fallas en el hogar de la caldera con una detección por sucesos de hasta 20 nanosegundos proporcionados por el hardware de la tarjeta. Asimismo, la representación cualitativa del modelado basado en redes de Petri, lo hace fácilmente extensible a un conjunto de fallas tan grande como se pretenda alcanzar o bien, fácilmente aplicable a los distintos niveles de operación del proceso en el generador de vapor, con el único requisito de reajustar los umbrales de detección por medio del software de programación ISE Foundation de Xilinxs.

Figura 7. Simulación realizada en el software ISE Fundation del SDDF en el hogar.

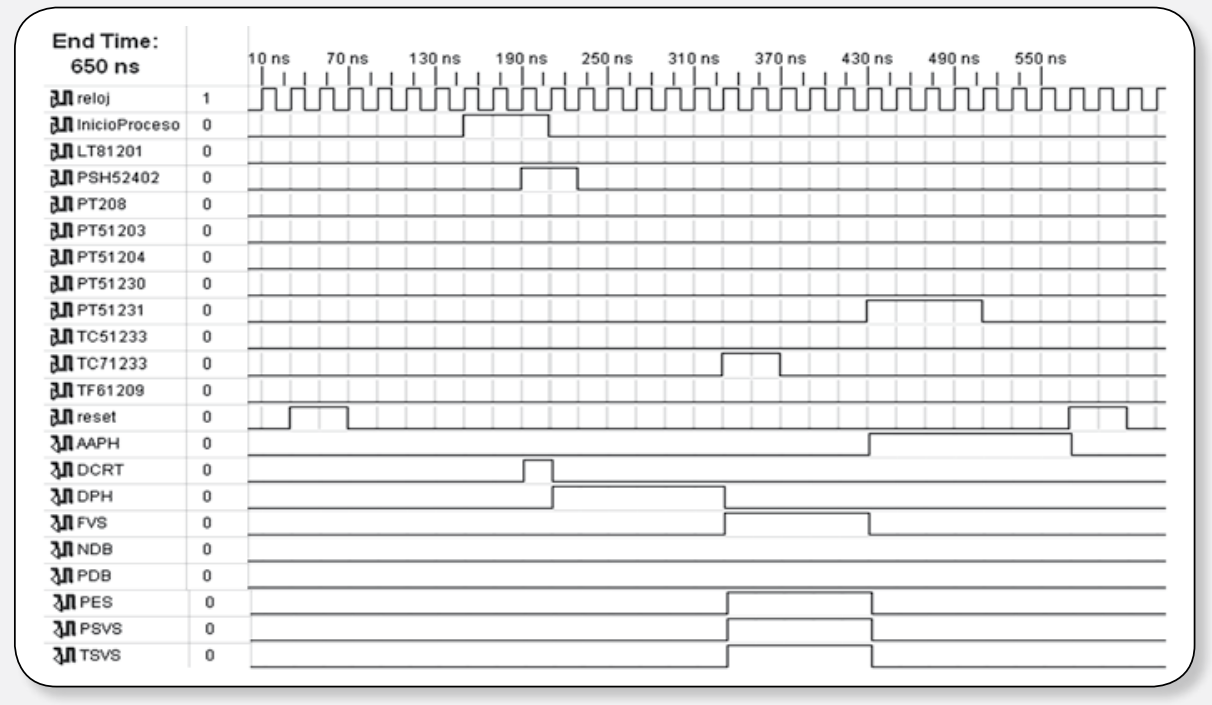


En este trabajo de investigación se abordó el tema de detección y diagnóstico de fallos mediante redes de Petri, por medio de un algoritmo sistemático implementado para la construcción del modelo. En él se pueden detectar el fallo de manera independiente y permite modificaciones o adiciones en el proceso (flexibilidad y propone la revisión On-line del proceso ya que la tarjeta cuenta con esta prestación). El algoritmo se aplica a un proceso de Hogar en la generación de vapor de la Termoeléctrica de Termotasajero Colgener S.a. E.s.p., el cual fue simulado utilizando la toolbox de Matlab PNTool, simulando la aplicación en Test Bench WaveFrom del software ISE Foundation de Xilinxs e implementado en el FPGA Spartan 2E de Xilinxs. Permitiendo a trabajo futuro hacer la extensión al diagnóstico de fallos a sistemas híbridos: petri-fuzzy, redes neuronales - redes de petri.

\section{Agradecimientos}

Los autores expresan sus agradecimientos a la empresa Termotasajero Colgener S.a. E.s.p. y a la Universidad de Pamplona, igualmente por la colaboración y asesoría se agradece al grupo REYCON y a los ingenieros Diego Fernando Feged Veles y Gerson Rodríguez, Lenin Salazar y a los técnicos Leivi Maldonado y Lorenzo Arias.

\section{Referencias}

\section{0}

\section{bibliográficas}

[1] Suárez C., D. A. Alfredo Sánchez L, J. E. Martínez P. y García B., C. D. (2006). Diagnóstico de fallas en el generador de vapor de una termoeléctrica usando redes de Petri.

[2] Correcher, A., Garcia, E., Morant, F. and Quiles, E., (2005). "Diagnóstico de Fallos Intermitentes: Un enfoque basado en modelos de eventos discretos"
Revista Iberoamericana de Automática e Informática.

[3] García, E., (2000) "Descomposición Modular de Diagnosticadores de Fallos Basados en modelos de eventos discretos" Tesis Doctoral.

[4] Sampath, M., Sengupta, R., Lafortune, S., Sinnamohidee, K. and Teneketzis, D., (1996) "Failure diagnosis using discrete event models" IEEE Trans On Contr. Systems , Vol. 4, no 2, pp. 105 124.

[5] Cheng, S.and Jeng, M. (2003). "Failure Diagnosis: A case Study on Modeling and Analysis by Petri Nets", IEEE.

[6] Genc, S. and Lafortune, S., (2006) "Distributed Diagnosis of Placesboundered Petri Nets", Department of Electrical Engineering and Computer Science, University of Michigan, USA.

[7] Giua, A. and Seatzu, C., (2005). "Fault detection for discrete event systems using Petri Nets with unobservable transitions", 44th IEEE Conference on Decision and Control, Seville, Spain.

[8] Ruíz, E., Jiménez, I., Ramírez, A. and López, E., (2004)"Fault Detection and Location in DES using Petri Nets", Proceedings of the 2004 IEEE, International Conference on Robotics \& Automation, New Orleans, LA.

[9] Trigos, M., García, E., Rodríguez, L., (2008) "Modelado y Diagnóstico de Fallos por Medio de Redes De Petri de un Sistema de Envasado de Líquidos" .

[10] Lo, K.L., H. S. Ng y J. Trecat (1997). Power systems fault diagnosis using Petri nets. IEE Proceedings of Generation, Transmition and. Distribution, IEE proceedings online, Vol. 144, pp. 231 236. 